\title{
MODELAMIENTO Y EVALUACIÓN DEL NIVEL DE CALIDAD DEL AIRE MEDIANTE EL ANÁLISIS DE GREY CLUSTERING, ESTUDIO DE CASO LIMA METROPOLITANA
}

\author{
MODELING AND AIR QUALITY ASSESSMENT THROUGH GREY \\ CLUSTERING ANALYSIS, CASE STUDY: LIMA METROPOLITANA
}

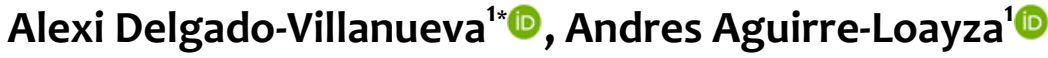

\author{
${ }^{1}$ Facultad de Ingeniería Ambiental, Universidad Nacional de Ingeniería, Lima, Perú. \\ Recibido (Recieved): 06/12/2018 Aceptado (Accepted): 02/03/2020
}

\begin{abstract}
RESUMEN
Actualmente los parámetros que evalúan la calidad del aire son analizados de manera independiente, dejando de lado el enfoque sistémico del ambiente, el cual establece que la calidad del aire está influenciada y controlada por muchos tipos de factores, en donde varios parámetros interactúan y se restringen mutuamente, por estas razones se propone un sistema de evaluación superior que tome en cuenta el alto grado de incertidumbre presente en el medio, denominada la metodología de análisis de Grey Clustering cuya base es la lógica difusa. La propuesta metodológica evaluará el nivel de calidad de aire en Lima Metropolitana, mediante el método de “Triangulación del punto medio en base a funciones de Whitenización - CTWF”, en donde se demostrará que el modelo propuesto es exacto, comparable y aplicable. Los datos de calidad del aire se obtuvieron del Servicio Nacional de Meteorología e Hidrología del Perú-Senamhi. El método CTWF se aplicó utilizando los principales indicadores de la calidad del aire, tales como, PM10, PM2.5, SO2 y No2. Los datos de muestreo se clasificaron usando el Índice de Calidad del Aire Anual -YACAQI; como resultado de la evaluación de la calidad del aire se determinó que la mayoría de distritos presentaban problemas muy graves de contaminación del aire. El diagnóstico permitirá que la sociedad en general y municipalidades tengan como resultado un instrumento técnico de análisis objetivo e integral, que brinda resultados fáciles de interpretar y permita enfocar los principales contaminantes persistentes en el medio.
\end{abstract}

Palabras Clave: evaluación, calidad del aire, enfoque sistémico, Grey Clustering

\section{ABSTRACT}

Currently, the criteria to air quality assessment are analysed independently, leaving aside the systemic approach of the environment, where air quality is influenced and controlled by many types of factors, where several parameters interact and mutually restrict each other for that reason a higher evaluation system is proposed by Grey Clustering analysis based on fuzzy logic, which takes into account the high degree of uncertainty present in the environment. The methodological proposal will assess the quality of air in Lima Metropolitana. In this work, we apply Center-point triangular whitenization weight functions (CTWF) method, where it will be demonstrated that the proposed model is exact, comparable and applicable. The monitoring data on each city of Lima Metropolitana were obtained from National Service of Meteorology and Hydrology of Peru Senamhi. The CTWF method was applied using parameters of air quality such as PM10, PM2.5, SO2 y No2. Then, the results were ranked using the Year Average Common Air Quality Index (YACAQI). Consequently, the results showed that most of the cities are polluted. Finally, the results of this study could be used by local authorities or central government to make the best decision to focus on the main persistent pollutants in the environment.

Keywords: assessment, air quality, system, Grey Clustering

\footnotetext{
* Corresponding author.:

E-mail: alexidelgado@hotmail.com
} 


\section{INTRODUCCION}

La atmósfera ha sufrido grandes cambios en composición y propiedad a lo largo de los años, desarrollándose procesos de transporte y dilución, así como reacciones químicas de transformación de sus contaminantes. La atmósfera es un medio que interactúa con diversos problemas ambientales derivados de determinadas sustancias presentes en el aire [1]. Las sustancias básicas que generan más del 90\% de la contaminación atmosférica se pueden resumir principalmente en cinco grupos: partículas en suspensión, óxidos de nitrógeno, óxidos de azufre y sulfatos, óxidos de carbono e hidrocarburos [2].

La evaluación de la calidad del aire, toma como indicadores de la calidad del aire los principales contaminantes atmosféricos, los cuáles son analizados manera independiente, dejando de lado el enfoque sistémico del ambiente, en donde cada uno de sus elementos interactúan y se restringen entre sí [3], por tal razón existe la necesidad de llevar este enfoque a la práctica de tal forma que la evaluación que se realice sea de objetiva e integral, evitando la subjetividad al establecer menor o mayor importancia en peso a alguno de estos indicadores ambientales.

El presente trabajo de investigación propone utilizar la metodología de análisis de Grey Clustering [4], la cual se puede aplicar por incidencia grey de matrices o funciones de whitenizacion. En este trabajo, se aplicó la "Triangulación del punto medio en base a funciones de Whitenización - CTWF", ya que la CTWF se aplica principalmente para probar si los objetos de observación pertenecen a clases predeterminadas, conocidas como grey clases [5], tal como se evidencia en los estudios de selección de estrategias innovadoras [6] y de evaluación de la calidad del aire por incidencia grey [7]. A diferencia de métodos estadísticos tradicionales, esta metodología considera la incertidumbre dentro de su análisis, al realizar la integración de los indicadores en evaluación; y considerando que la contaminación ambiental es un problema con alta incertidumbre debido a las distintas fuentes de contaminación y condiciones meteorológicas, su aplicación es la más adecuada [8].

\section{METODOLOGÍA}

\subsection{METODOLOGÍA DE ANÁLISIS GREY CLUSTERING}

Se explica el método CTWF, el cual se desarrolló para realizar la evaluación de la calidad del aire en 10 distritos de Lima Metropolitana. Asumiendo que existen $m$ puntos de monitoreo, $n$ parámetros de calidad del aire y s clasificaciones grey, según los valores de monitoreo de calidad del aire $x_{i j}=(1,2, \ldots, \mathrm{n}$; $j=1,2, \ldots, m)$, se puede realizar la clasificación grey, llamada Grey Clustering siguiendo los pasos mostrados a continuación [8], [9]:

Paso 1: Se realiza el adimensionamiento de los datos de monitoreo de calidad del aire de cada indicador ambiental (i) y de los parámetros del Índice de Calidad del Aire (j) seleccionados.

Paso 2: Establecer la clasificación grey a partir del índice de calidad ambiental mediante el método CTWF, tal como se muestra en la Fig. 1.

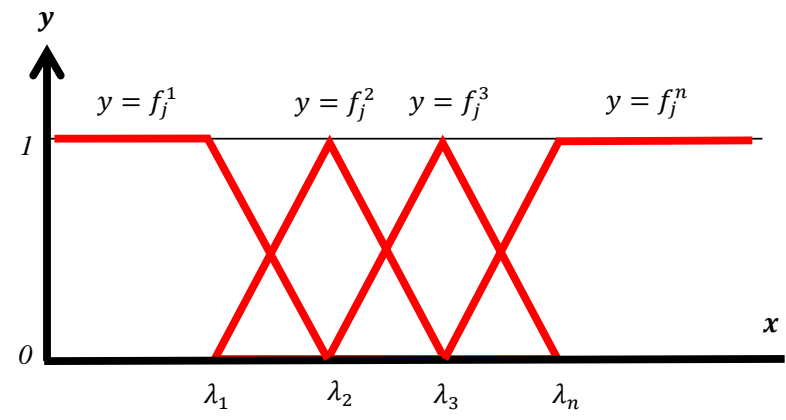

Fig. 1. CTWF en relación al Índice Anual de Calidad Ambiental [8]

Donde:

$$
\begin{aligned}
& y=f_{j}^{1}=\text { Muy buena calidad } \\
& y=f_{j}^{2}=\text { Buena calidad } \\
& y=f_{j}^{3}=\text { Mala calidad } \\
& y=f_{j}^{4}=\text { Muy mala calidad }
\end{aligned}
$$

Una vez establecida la clasificación grey mediante el método CTWF estas se calculan a partir de la kth clase grey, $k=1,2,3,4$, del jth parámetro, $j=1,2, \ldots n$, para un valor del monitoreo de la calidad del aire $x_{i j}$ según las ecuaciones (1), (2) y (3).

$$
\begin{aligned}
& f_{j}^{1}\left(x_{i j}\right)=\left\{\begin{array}{cc}
1, & x \in\left[0, \lambda_{j}^{1}\right] \\
\frac{\lambda_{j}^{2}-x}{\lambda_{j}^{2}-\lambda_{j}^{1}}, & x \in] \lambda_{j}^{1}, \lambda_{j}^{2}[ \\
0, & x \in\left[\lambda_{j}^{2},+\infty[\right.
\end{array}\right. \\
& f_{j}^{k}\left(x_{i j}\right)= \begin{cases}\frac{x-\lambda_{j}^{k-1}}{\lambda_{j}^{k}-\lambda_{j}^{k-1}}, & \left.x \in] \lambda_{j}^{k-1}, \lambda_{j}^{k}\right] \\
\frac{\lambda_{j}^{k+1}-x}{\lambda_{j}^{k+1}-\lambda_{j}^{k}}, & x \in] \lambda_{j}^{k}, \lambda_{j}^{k+1}[ \\
0, \quad x \in\left[0, \lambda_{j}^{k-1}\right] \cup\left[\lambda_{j}^{k+1},+\infty[\right.\end{cases} \\
& f_{j}^{4}\left(x_{i j}\right)=\left\{\begin{array}{cc}
\frac{x-\lambda_{j}^{3}}{\lambda_{j}^{4}-\lambda_{j}^{3}}, & x \in] \lambda_{j}^{3}, \lambda_{j}^{4}[ \\
1, & x \in\left[\lambda_{j}^{4},+\infty[\right. \\
0, & x \in\left[0, \lambda_{j}^{3}\right]
\end{array}\right.
\end{aligned}
$$


Paso 3: Calcular el peso de cada parámetro del índice de calidad ambiental de manera objetiva, conocido como peso clustering $n_{i j}$, el cual se calcular según la ecuación (4).

$$
n_{j}^{k}=\frac{1 / \lambda_{j}^{k}}{\sum_{j=1}^{m} 1 / \lambda_{j}^{k}}
$$

Paso 4: Una vez obtenido los valores evaluados en las funciones de whitenizacion y los pesos de cada parámetro, se procede a calcular el coeficiente de clustering para cada valor obtenido en las distintas clasificaciones de grey mediante la ecuación (5).

$$
\sigma_{i}^{k}=\sum_{j=1}^{n} f_{j}^{k}\left(x_{i j}\right) \cdot n_{j}
$$

Paso 5: $\quad$ Se establece que el $\max _{1 \leq k \leq s}\left\{\sigma_{i}^{k}\right\}=\sigma_{i}^{k^{*}}$, define la clasificación de la calidad del aire del distrito evaluado.

\section{ESTUDIO DEL CASO}

La zona de estudio es Lima Metropolitana, ya que se busca evaluar la calidad del aire en el año 2017, en base a todas las Estaciones de Monitoreo de Calidad del Aire existentes - EMA. Según información proporcionada por el Servicio Nacional de Meteorológica e Hidrología del Perú - Senamhi, Lima Metropolitana cuenta con un total de 10 Estaciones de Monitoreo de Calidad del Aire en todos sus distritos

El área total de Lima Metropolitana es de $2,819 \mathrm{~km}^{2}$ y el área total de todos los distritos que cuentan con una estación de calidad del aire es de 839 km², lo cual representa un $29.7 \%$ del área en estudio.
En la Fig. 2 se observa los distritos de Lima Metropolitana y aquellas que cuentan con estaciones de calidad del aire.

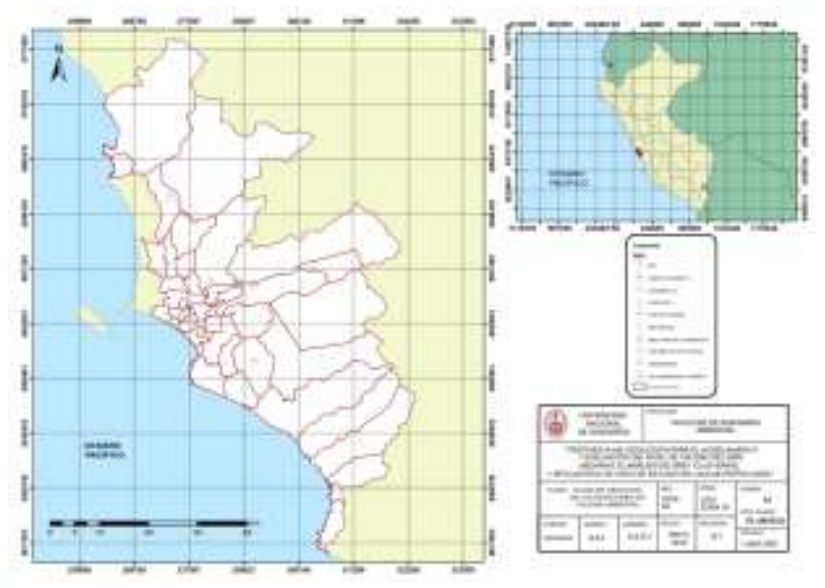

Fig. 2. Ubicación de las Estaciones de Calidad del Aire en Lima Metropolitana

En la Tabla I se muestra las coordenadas y códigos de las estaciones de monitoreo de la calidad del aire que maneja el Senamhi [10], así como las abreviaciones utilizadas para cada distrito que cuenta con una de estas estaciones.

\subsection{APLICACIÓN DEL MÉTODO CTWF}

El cálculo usado para el estudio del caso se basa en el método CTWF, mediante los procedimientos mostrados:

Paso 1: Se establece el estándar e Índice de Calidad Ambiental y los datos de calidad de aire a clasificar.

La Organización Mundial de Salud define los parámetros internacionales que no deberían de excederse para evitar un impacto negativo en la salud humana, la vegetación, los edificios, monumentos, sitios de patrimonio histórico, etc. Las ciudades u otras entidades internacionales igualmente pueden definir

TABLA I

\begin{tabular}{|c|c|c|c|c|c|}
\hline \multirow{2}{*}{$\begin{array}{c}\text { Estaciones de } \\
\text { Monitoreo }\end{array}$} & \multirow[b]{2}{*}{ Código } & \multicolumn{2}{|c|}{ Coordenadas WGS 84} & \multirow[b]{2}{*}{ Distrito } & \multirow[b]{2}{*}{ Abreviación } \\
\hline & & Longitud & Latitud & & \\
\hline Ate Vitarte & 112192 & 291125.97 & 8669830.18 & Ate Vitarte & ATE \\
\hline Huachipa & 112266 & 287828.94 & 8670606.07 & Huachipa & HUA \\
\hline San Juan de Lurigancho & 112267 & 282383.4 & 8670566.99 & San Juan de Lurigancho & SJL \\
\hline Santa Anita & 112208 & 291125.97 & 8669830.18 & Santa Anita & SA \\
\hline Puente Piedra & 111287 & 274053.08 & 8687726.51 & Puente Piedra & PP \\
\hline San Borja & 112193 & 281483.19 & 8660631.11 & San Borja & SB \\
\hline Campo de Marte & 112194 & 277597.42 & 8664818.03 & Jesús María & $J M$ \\
\hline Carabayllo & 111286 & 278497.59 & 8683450.49 & Carabayllo & CAR \\
\hline Villa María del Triunfo & 112233 & 291083.74 & 8654309.37 & Villa María del Triunfo & VMT \\
\hline San Martín de Porres & 112265 & 273047.65 & 8671604.21 & San Martín de Porres & SMP \\
\hline
\end{tabular}

Coordenadas de las estaciones de monitoreo de Calidad del Aire

Nota: Estaciones de monitoreo de la calidad del aire operadas por Senamhi. [10]. 
parámetros locales como límites para no excederlos siempre y cuando exista un sustento adecuado. En este caso, se tomaron los límites proporcionados por la Unión Europea (UE), ya que brinda un índice para datos de calidad del aire anuales.

Al igual que el índice horario y diario, el Índice de Calidad del Aire promedio Anual (YACAQI) es calculado para gestiones en sitios urbanizados de la ciudad cuando existe una carencia de datos. Esto implica que los índices resultantes no necesariamente reflejan una imagen completa y equilibrada de una ciudad. Los índices de los indicadores de Calidad Ambiental se calculan como se muestra en la Tabla II [11].

TABLA II

Esquema para calcular el Índice de Calidad del Aire Anual

\begin{tabular}{|c|c|c|}
\hline $\begin{array}{c}\text { Indicador de Calidad } \\
\text { Ambiental } \\
\end{array}$ & $\begin{array}{c}\text { Estándar de Calidad } \\
\text { Ambiental }\end{array}$ & $\begin{array}{l}\text { Cálculo del Índice de } \\
\text { Calidad Ambiental }\end{array}$ \\
\hline $\mathrm{NO}_{2}$ & $\begin{array}{c}\text { Promedio anual } \\
\mathbf{4 0} \boldsymbol{\mu g} / \mathbf{m}^{3}\end{array}$ & Year average/40 \\
\hline$P M_{10}$ & $\begin{array}{l}\text { Promedio anual } \\
\qquad \mathbf{4 0} \boldsymbol{\mu g} / \mathbf{m}^{3}\end{array}$ & Year average/40 \\
\hline $\mathrm{SO}_{2}$ & $\begin{array}{l}\text { Promedio anual } \\
\qquad 20_{\mu \mathrm{g} / \mathbf{m}^{3}}\end{array}$ & Year average/20 \\
\hline$P M_{2.5}$ & $\begin{array}{l}\text { Promedio anual } \\
\qquad \mathbf{2 0} \mu \mathrm{g} / \mathbf{m}^{3}\end{array}$ & Year average/20 \\
\hline
\end{tabular}

Nota: ECA del PM10, PM2.5, SO2 y No2 según la UE. [11].

En la Tabla III se observa la clasificación del nivel de contaminación en base a la estandarización del índice de calidad ambiental propuesto por la UE: Muy buena calidad ( $\left.\lambda_{1}\right)$, Buena calidad ( $\left.\lambda_{2}\right)$, Mala calidad ( $\left.\lambda_{3}\right)$, Muy mala calidad $\left(\lambda_{4}\right)$.

TABLA III

Datos estándares del Índice de Calidad Ambiental propuestos por la UE

\begin{tabular}{ccccc}
\hline Clase & $\lambda_{1}$ & $\lambda_{2}$ & $\lambda_{3}$ & $\lambda_{4}$ \\
$\mathrm{PM} 2.5$ & $0-50$ & $50-100$ & $100-150$ & $>150$ \\
$\mathrm{PM} 10$ & $0-50$ & $50-100$ & $100-150$ & $>150$ \\
$\mathrm{NO} 2$ & $0-50$ & $50-100$ & $100-150$ & $>150$ \\
$\mathrm{SO} 2$ & $0-50$ & $50-100$ & $100-150$ & $>150$ \\
\hline
\end{tabular}

Nota: Estandarización de los ECA propuestos por la UE.

Al conocer los valores que propone la UE, se busca el punto central de los intervalos y posteriormente se adimensionan los parámetros para poder realizar los cálculos que la metodología del Análisis de Grey Clustering exige, resultado de eso se observa en la Tabla IV.
TABLA IV

Datos estándares adimensionados del Índice de Calidad Ambiental propuesto por la UE

\begin{tabular}{ccccc}
\hline Clase & $\lambda 1$ & $\lambda 2$ & $\lambda_{3}$ & $\lambda 4$ \\
PM 2.5 & 0.250 & 0.750 & 1.250 & 1.750 \\
PM 10 & 0.250 & 0.750 & 1.250 & 1.750 \\
$\mathrm{NO}_{2}$ & 0.250 & 0.750 & 1.250 & 1.750 \\
$\mathrm{SO}_{2}$ & 0.250 & 0.750 & 1.250 & 1.750 \\
\hline
\end{tabular}

Nota: Adimensionamiento de los Estándares del YACAQI propuestos por la UE.

En la Tabla $V$ se presenta el promedio anual correspondiente al año 2017, de los datos de monitoreo de la calidad del aire en 10 estaciones de monitoreo de calidad del aire del Senamhi [10].

TABLA V

Datos del monitoreo de calidad del aire

\begin{tabular}{ccccc}
\hline Distritos & PM 2.5 & PM 10 & NO2 & SO2 \\
SMP & 17.55 & 51.91 & 16.60 & 6.16 \\
SB & 17.69 & 51.22 & 26.24 & 11.69 \\
SA & 30.73 & 76.78 & 26.40 & 8.88 \\
ATE & 42.35 & 118.62 & 42.97 & 49.79 \\
CM & 13.20 & 39.38 & 17.13 & 7.69 \\
CAR & - & 81.93 & 26.41 & 4.78 \\
HUA & 33.66 & 110.57 & 40.98 & 12.97 \\
PP & 30.42 & 110.07 & 24.81 & 8.24 \\
SJL & 32.69 & 91.76 & 37.47 & 11.46 \\
VMT & 25.50 & 132.44 & 14.11 & 6.35 \\
\hline
\end{tabular}

Nota: Resultados del monitoreo de las estaciones de calidad del aire durante el año 2017, información Senamhi. [10]

En la Tabla VI se presenta los datos adimensionados del monitoreo de la calidad del aire, estos parámetros son aquellos que serán evaluados y clasificados el Índice de Calidad del Aire promedio Anual propuesto por la UE.

TABLA VI

Datos adimensionados del monitoreo de calidad del aire

\begin{tabular}{ccccc}
\hline Distritos & PM 2.5 & PM 10 & NO2 & SO2 \\
SMP & 0.88 & 1.30 & 0.42 & 0.31 \\
SB & 0.88 & 1.28 & 0.66 & 0.58 \\
SA & 1.54 & 1.92 & 0.66 & 0.44 \\
ATE & 2.12 & 2.97 & 1.07 & 2.49 \\
CM & 0.66 & 0.98 & 0.43 & 0.38 \\
CAR & 0.00 & 2.05 & 0.66 & 0.24 \\
HUA & 1.68 & 2.76 & 1.02 & 0.65 \\
PP & 1.52 & 2.75 & 0.62 & 0.41 \\
SJL & 1.63 & 2.29 & 0.94 & 0.57 \\
VMT & 1.28 & 3.31 & 0.35 & 0.32 \\
\hline
\end{tabular}

Nota: Se adimensionó los resultados del monitoreo de calidad del aire durante el año 2017 tal como se especifica en la metodología.

Paso 2: Los valores de la Tabla IV se sustituyeron en las ecuaciones (1), (2) y (3), con la finalidad de obtener las funciones de whitenizacion en las 4 clasificaciones grey. 
Posteriormente se evaluó los datos de la Tabla VI en las funciones de whitenizacion, dando como resultado los valores observados en las Tablas VIII, IX, X, XI, XII, XIII, XIV, XV, XVI y XVII.

Paso 3: Tomando como base los valores de la Tabla IV, se determinó los valores de la Tabla VII, la cual muestra los pesos clustering $\left(n_{i j}\right)$ de cada parámetro del Índice de Calidad Ambiental propuesto por la UE mediante el uso de la ecuación (4).

TABLA VII

Pesos Clustering para cada parámetro del YACAQI

\begin{tabular}{ccccc}
\hline Clase & $\lambda 1$ & $\lambda 2$ & $\lambda 3$ & $\lambda 4$ \\
PM 2.5 & 0.250 & 0.250 & 0.250 & 0.250 \\
PM 10 & 0.250 & 0.250 & 0.250 & 0.250 \\
$\mathrm{NO}_{2}$ & 0.250 & 0.250 & 0.250 & 0.250 \\
$\mathrm{SO}_{2}$ & 0.250 & 0.250 & 0.250 & 0.250 \\
\hline
\end{tabular}

Nota: Los pesos clustering se determinaron según la metodología propuesta.

Paso 4: En las Tablas VIII, IX, X, XI, XII, XIII, XIV, XV, XVI y XVII.

Se observa el resultado de calcular los coeficientes de cluster $\left(\sigma_{i}^{k}\right)$ para cada punto de monitoreo ubicado en 10 distritos diferentes, mediante el uso ecuación (5).

TABLA VIII

Función de Whitenización evaluada para San Martín de Porres

\begin{tabular}{lccccc}
\hline $\mathrm{SMP}$ & $\mathrm{PM} 2.5$ & $\mathrm{PM} \mathrm{10}$ & $\mathrm{NO} 2$ & $\mathrm{SO} 2$ & $\boldsymbol{\sigma}_{\boldsymbol{i}}^{\boldsymbol{k}}$ \\
$\boldsymbol{f}_{\boldsymbol{j}}^{\mathbf{1}}(\boldsymbol{x})$ & 0 & 0 & 0.670 & 0.884 & 0.389 \\
$\boldsymbol{f}_{\boldsymbol{j}}^{\mathbf{2}}(\boldsymbol{x})$ & 0.745 & 0 & 0.330 & 0.116 & 0.298 \\
$\boldsymbol{f}_{\boldsymbol{j}}^{\mathbf{3}}(\boldsymbol{x})$ & 0.255 & 0.905 & 0 & 0 & 0.290 \\
$\boldsymbol{f}_{\boldsymbol{j}}^{\boldsymbol{4}}(\boldsymbol{x})$ & 0 & 0.095 & 0 & 0 & 0.024
\end{tabular}

Nota: Se calculó los coeficientes de cluster según las funciones de whitenización del PM10, PM2.5, SO2 y No2 en SMP.

TABLA IX

Función de Whitenización evaluada para San Borja

\begin{tabular}{cccccc}
\hline $\mathrm{SB}$ & $\mathrm{PM} 2.5$ & $\mathrm{PM} \mathrm{10}$ & $\mathrm{NO} 2$ & $\mathrm{SO} 2$ & $\boldsymbol{\sigma}_{\boldsymbol{i}}^{\boldsymbol{k}}$ \\
$\boldsymbol{f}_{\boldsymbol{j}}^{\mathbf{1}}(\boldsymbol{x})$ & 0 & 0 & 0.188 & 0.331 & 0.130 \\
$\boldsymbol{f}_{\boldsymbol{j}}^{\mathbf{2}}(\boldsymbol{x})$ & 0.731 & 0 & 0.812 & 0.669 & 0.553 \\
$\boldsymbol{f}_{\boldsymbol{j}}^{3}(\boldsymbol{x})$ & 0.269 & 0.939 & 0 & 0 & 0.302 \\
$\boldsymbol{f}_{\boldsymbol{j}}^{\mathbf{4}}(\boldsymbol{x})$ & 0 & 0.061 & 0 & 0 & 0.015 \\
\hline
\end{tabular}

Nota: Se calculó los coeficientes de cluster según las funciones de whitenización del PM10, PM2.5, SO2 y NO2 en SB.

TABLAX

Función de Whitenización evaluada para Santa Anita

\begin{tabular}{cccccc}
\hline $\mathrm{SA}$ & $\mathrm{PM} \mathrm{2.5}$ & $\mathrm{PM} \mathrm{10}$ & $\mathrm{NO} 2$ & $\mathrm{SO} 2$ & $\boldsymbol{\sigma}_{\boldsymbol{i}}^{\boldsymbol{k}}$ \\
$\boldsymbol{f}_{\boldsymbol{j}}^{\mathbf{1}}(\boldsymbol{x})$ & 0 & 0 & 0.180 & 0.612 & 0.198 \\
$\boldsymbol{f}_{\boldsymbol{j}}^{\mathbf{2}}(\boldsymbol{x})$ & 0 & 0 & 0.820 & 0.388 & 0.302 \\
$\boldsymbol{f}_{\boldsymbol{j}}^{\mathbf{3}}(\boldsymbol{x})$ & 0.427 & 0 & 0 & 0 & 0.107 \\
$\boldsymbol{f}_{\boldsymbol{j}}^{\mathbf{4}}(\boldsymbol{x})$ & 0.573 & 1 & 0 & 0 & 0.393 \\
\hline
\end{tabular}

Nota: Se calculó los coeficientes de cluster según las funciones de whitenización del PM10, PM2.5, SO2 y No2 en SA.
TABLA XI

Función de Whitenización evaluada para Ate

\begin{tabular}{lccccc}
\hline $\mathrm{ATE}$ & $\mathrm{PM} \mathrm{2.5}$ & $\mathrm{PM} \mathrm{10}$ & $\mathrm{NO} 2$ & $\mathrm{SO} 2$ & $\boldsymbol{\sigma}_{\boldsymbol{i}}^{\boldsymbol{k}}$ \\
$\boldsymbol{f}_{\boldsymbol{j}}^{\mathbf{1}}(\boldsymbol{x})$ & 0 & 0 & 0 & 0 & 0 \\
$\boldsymbol{f}_{\boldsymbol{j}}^{2}(\boldsymbol{x})$ & 0 & 0 & 0.351 & 0 & 0.088 \\
$\boldsymbol{f}_{\boldsymbol{j}}^{3}(\boldsymbol{x})$ & 0 & 0 & 0.649 & 0 & 0.162 \\
$\boldsymbol{f}_{\boldsymbol{j}}^{\mathbf{4}}(\boldsymbol{x})$ & 1 & 1 & 0 & 1 & 0.75
\end{tabular}

Nota: Se calculó los coeficientes de cluster según las funciones de whitenización del PM10, PM2.5, SO2 y No2 en ATE.

TABLA XII

Función de Whitenización evaluada para Jesús María - Campo de Marte

\begin{tabular}{cccccc}
\hline JM & $\mathrm{PM} \mathrm{2.5}$ & $\mathrm{PM} \mathrm{10}$ & $\mathrm{NO} 2$ & $\mathrm{SO} 2$ & $\boldsymbol{\sigma}_{\boldsymbol{i}}^{\boldsymbol{k}}$ \\
$\boldsymbol{f}_{\boldsymbol{j}}^{\mathbf{1}}(\boldsymbol{x})$ & 0.180 & 0 & 0.643 & 0.731 & 0.388 \\
$\boldsymbol{f}_{\boldsymbol{j}}^{\mathbf{2}}(\boldsymbol{x})$ & 0.820 & 0.531 & 0.357 & 0.269 & 0.494 \\
$\boldsymbol{f}_{\boldsymbol{j}}^{3}(\boldsymbol{x})$ & 0 & 0.469 & 0 & 0 & 0.117 \\
$\boldsymbol{f}_{\boldsymbol{j}}^{\boldsymbol{4}}(\boldsymbol{x})$ & 0 & 0 & 0 & 0 & 0 \\
\hline
\end{tabular}

Nota: Se calculó los coeficientes de cluster según las funciones de whitenización del PM10, PM2.5, SO2 y No2 en JM.

TABLA XIII

Función de Whitenización evaluada para Carabayllo

\begin{tabular}{lccccc}
\hline $\mathrm{CAR}$ & $\mathrm{PM} 2.5$ & $\mathrm{PM} 10$ & $\mathrm{NO} 2$ & $\mathrm{SO} 2$ & $\boldsymbol{\sigma}_{\boldsymbol{i}}^{\boldsymbol{k}}$ \\
$\boldsymbol{f}_{\boldsymbol{j}}^{\mathbf{1}}(\boldsymbol{x})$ & 1 & 0 & 0.180 & 1 & 0.545 \\
$\boldsymbol{f}_{\boldsymbol{j}}^{2}(\boldsymbol{x})$ & 0 & 0 & 0.820 & 0 & 0.205 \\
$\boldsymbol{f}_{\boldsymbol{j}}^{3}(\boldsymbol{x})$ & 0 & 0 & 0 & 0 & 0 \\
$\boldsymbol{f}_{\boldsymbol{j}}^{4}(\boldsymbol{x})$ & 0 & 1 & 0 & 0 & 0.250
\end{tabular}

Nota: Se calculó los coeficientes de cluster según las funciones de whitenización del PM10, PM2.5, SO2 y No2 en CAR.

TABLA XIV

Función de Whitenización evaluada para Huachipa

$\begin{array}{cccccc}\text { HUA } & \mathrm{PM} \mathrm{2.5} & \mathrm{PM} \mathrm{10} & \mathrm{NO} 2 & \mathrm{SO} 2 & \boldsymbol{\sigma}_{\boldsymbol{i}}^{\boldsymbol{k}} \\ \boldsymbol{f}_{\boldsymbol{j}}^{\mathbf{1}}(\boldsymbol{x}) & 0 & 0 & 0 & 0.203 & 0.051 \\ \boldsymbol{f}_{\boldsymbol{j}}^{2}(\boldsymbol{x}) & 0 & 0 & 0.451 & 0.797 & 0.312 \\ \boldsymbol{f}_{\boldsymbol{j}}^{\mathbf{3}}(\boldsymbol{x}) & 0.134 & 0 & 0.549 & 0 & 0.171 \\ \boldsymbol{f}_{\boldsymbol{j}}^{\mathbf{4}}(\boldsymbol{x}) & 0.866 & 1 & 0 & 0 & 0.466\end{array}$

Nota: Se calculó los coeficientes de cluster según las funciones de whitenización del PM10, PM2.5, SO2 y No2 en HUA.

TABLA XV

Función de Whitenización evaluada para Puente Piedra

\begin{tabular}{cccccc}
\hline $\mathrm{PP}$ & $\mathrm{PM} 2.5$ & $\mathrm{PM} \mathrm{10}$ & $\mathrm{NO} 2$ & $\mathrm{SO} 2$ & $\boldsymbol{\sigma}_{\boldsymbol{i}}^{\boldsymbol{k}}$ \\
$\boldsymbol{f}_{\boldsymbol{j}}^{\mathbf{1}}(\boldsymbol{x})$ & 0 & 0 & 0.260 & 0.676 & 0.234 \\
$\boldsymbol{f}_{\boldsymbol{j}}^{2}(\boldsymbol{x})$ & 0 & 0 & 0.740 & 0.324 & 0.266 \\
$\boldsymbol{f}_{\boldsymbol{j}}^{3}(\boldsymbol{x})$ & 0.458 & 0 & 0 & 0 & 0.114 \\
$\boldsymbol{f}_{\boldsymbol{j}}^{\mathbf{4}}(\boldsymbol{x})$ & 0.542 & 1 & 0 & 0 & 0.386
\end{tabular}

Nota: Se calculó los coeficientes de cluster según las funciones de whitenización del PM10, PM2.5, SO2 y No2 en PP. 
TABLA XVI

Función de Whitenización evaluada para San Juan de Lurigancho

$\begin{array}{lccccc}\text { SJL } & \mathrm{PM} 2.5 & \mathrm{PM} \mathrm{10} & \mathrm{NO} 2 & \mathrm{SO} 2 & \boldsymbol{\sigma}_{\boldsymbol{i}}^{\boldsymbol{k}} \\ \boldsymbol{f}_{\boldsymbol{j}}^{\mathbf{1}}(\boldsymbol{x}) & 0 & 0 & 0 & 0.354 & 0.089 \\ \boldsymbol{f}_{\boldsymbol{j}}^{2}(\boldsymbol{x}) & 0 & 0 & 0.626 & 0.646 & 0.318 \\ \boldsymbol{f}_{\boldsymbol{j}}^{\mathbf{3}}(\boldsymbol{x}) & 0.231 & 0 & 0.374 & 0 & 0.151 \\ \boldsymbol{f}_{\boldsymbol{j}}^{\mathbf{4}}(\boldsymbol{x}) & 0.769 & 1 & 0 & 0 & 0.442\end{array}$

Nota: Se calculó los coeficientes de cluster según las funciones de whitenización del PM10, PM2.5, SO2 y No2 en SJL.

TABLA XVII

Función de Whitenización evaluada para Villa María del Triunfo

\begin{tabular}{lccccc}
\hline $\mathrm{VMT}$ & $\mathrm{PM} 2.5$ & $\mathrm{PM} 10$ & $\mathrm{NO} 2$ & $\mathrm{SO} 2$ & $\boldsymbol{\sigma}_{\boldsymbol{i}}^{\boldsymbol{k}}$ \\
$\boldsymbol{f}_{\boldsymbol{j}}^{\mathbf{1}}(\boldsymbol{x})$ & 0 & 0 & 0.795 & 0.865 & 0.415 \\
$\boldsymbol{f}_{\boldsymbol{j}}^{\mathbf{2}}(\boldsymbol{x})$ & 0 & 0 & 0.205 & 0.135 & 0.085 \\
$\boldsymbol{f}_{\boldsymbol{j}}^{\mathbf{3}}(\boldsymbol{x})$ & 0.950 & 0 & 0 & 0 & 0.237 \\
$\boldsymbol{f}_{\boldsymbol{j}}^{\boldsymbol{4}}(\boldsymbol{x})$ & 0.050 & 1 & 0 & 0 & 0.263 \\
\hline
\end{tabular}

Nota: Se calculó los coeficientes de cluster según las funciones de whitenización del PM10, PM2.5, SO2 y No2 en VMT.

Paso 5: Por último se aplica la condición $\max _{1 \leq k \leq s}\left\{\sigma_{i}^{k}\right\}=\sigma_{i}^{k^{*}}$, la cual determina a que clase grey pertenece cada punto de monitoreo y que calidad del aire tiene cada distrito, los resultados se observan en la Tabla XVIII.

TABLA XVIII

Coeficientes de Cluster

\begin{tabular}{cccc}
\hline Distritos & Nombre & $\boldsymbol{\sigma}_{\boldsymbol{i}}^{\boldsymbol{k}}$ & YACAQI \\
ATE & Ate Vitarte & 0.750 & Muy mala Calidad \\
HUA & Huachipa & 0.466 & Muy mala Calidad \\
SJL & San Juan de & 0.442 & Muy mala Calidad \\
& Lurigancho & & \\
SA & Santa Anita & 0.393 & Muy mala Calidad \\
PP & Puente Piedra & 0.386 & Muy mala Calidad \\
SB & San Borja & 0.553 & Buena Calidad \\
JM & Campo de Marte & 0.494 & Buena Calidad \\
CAR & Carabayllo & 0.545 & Muy buena Calidad \\
VMT & Villa Maria del Triunfo & 0.415 & Muy buena Calidad \\
SMP & San Martín de Porres & 0.389 & Muy buena Calidad \\
\hline \multicolumn{2}{l}{ Nota: El } & máximo coeficiente cluster & de las funciones de \\
whitenización evaluadas determinó el resultado de la clasificación de \\
la calidad del aire.
\end{tabular}

\section{RESULTADOS Y DISCUSIONES}

El resultado de la evaluación de la calidad del aire de los 10 distritos nos permite realizar una jerarquía de la calidad de aire según el índice propuesto por la Unión Europea, la cual se muestra en una relación de mayor calidad a menor calidad del aire:

$$
\begin{gathered}
\mathrm{ATE}>\mathrm{HUA}>\mathrm{SJL}>\mathrm{SA}>\mathrm{PP}>\mathrm{SB}>\mathrm{CM}>\mathrm{CAR}>\mathrm{VMT}> \\
\mathrm{SMP}
\end{gathered}
$$

En donde el distrito con mayor contaminación atmosférica de Lima Metropolitana para el año 2017 es Ate Vitarte y el menos contaminado, el distrito de San Martín de Porres.
En el caso de Carabayllo la información obtenida no es precisa, ya que al no registrar datos de PM 2.5 en su estación respectiva, se considera una generación de PM $2.5=0 \mu g \mathrm{~m}^{\wedge} 3$ lo cual interfiere y hace que se obtenga como resultado una muy buena calidad del aire, por esta razón no se toma en cuenta para una interpretación general de la situación actual de la calidad del aire en Lima Metropolitana.

En base a estos resultados de las estaciones de calidad del aire se puede inferir que la mayor cantidad del territorio de Lima Metropolitano tiene problemas de calidad del aire, los cuales llegan a ser muy serios por presentar una muy mala calidad de esta.

\section{CONCLUSIONES}

- La metodología de Análisis de Grey Clustering determino la calidad del aire en 10 distritos de Lima metropolitana mediante una clasificación adecuada de cada parámetro y una posterior integración de estos; además de considerar la incertidumbre presente en el ambiente en el desarrollo de su análisis, a diferencia de otros métodos como el estadístico y los propuestos por la Environment Protecting Agency-EPA.

- $\quad$ El diagnóstico permitirá que la sociedad en general y municipalidades tengan como resultado un instrumento técnico de análisis objetivo e integral, que brinde resultados fáciles de interpretar y permita enfocar los principales contaminantes persistentes en el medioambiente a nivel nacional, regional y local.

- $\quad$ Los resultados obtenidos proyectan presentarse al Senamhi, con la finalidad de desarrollar un instrumento para la gestión de la Calidad del Aire que permita su protección y vigilancia.

\section{AGRADECIMIENTOS}

Agradecemos a la Facultad de Ingeniería Ambiental de la Universidad Nacional de Ingeniería, Lima, Perú, por financiar este proyecto, como parte del programa de investigación formativa 2018.

\section{REFERENCIAS}

[1] A. Gallego Picó, I. González Fernández, B. Sánchez Gimeno, P. Fernández Hernando, R.M. Garcinuño Martínez, J.C. Bravo, J.A. Pradana, M. A. García Mayor y J. S. Durand Alegría. Contaminación Atmosférica. Primera edición. Madrid: U.N.E.D.; 2012. 441 p.

[2] A. R. Guerrero Ruiz, E. Ortega Cantero y M. L. Rojas Cervantes. Origen y control de los contaminantes. 2011. $406 \mathrm{p}$.

[3] O. R. Sánchez-Ccoyllo y C. G. Ordóñez Aquino. Evaluación de la calidad del aire en Lima Metropolitana 2015, 2016.

[4] A. Delgado and I. Romero. "Applying Grey Systems and Shannon Entropy to Social Impact Assessment and Environmental Conflict Analysis", International Journal of Applied Engineering Research, vol. 12, no. 24, pp. 14327-14337, 2017. Accessed: March 7, 2018. [Online]. Available: http://www.ripublication.com/ijaer17/ijaerv12n24_47.pdf 
[5] A. Delgado, A. Aguirre, E. Palomino and G. Salazar, "Applying triangular whitenization weight functions to assess water quality of main affluents of Rimac river", in Proc 2017 Electron Congr. E-CON UNI, Lima.

[6] Y. Zhang, J. Ni, J. Liu and L. Jian. "Grey evaluation empirical study based on center-point triangular whitenization weight function of Jiangsu Province industrial technology innovation strategy alliance", Grey Systems: Theory and Application, vol. 4, no. 1, pp. 124-136, 2014.

[7] W. Meng, B. Zeng B, S. F. Liu and N. M. Xie, "A grey incidence evaluation on air quality", in Proc IEEE Int Conf Grey Syst Intell Serv GSIS, 2013, pp. 49-52.

[8] S. Liu and Y. Lin, Grey Systems: Theory and Applications. Berlin: Springer; 2010.

[9] A. Delgado and I. Romero, "Environmental conflict analysis using an integrated grey clustering and entropy-weight method: A case study of a mining project in Peru", Environmental Modelling \& Software, vol. 77, pp. 108-121, 2016. [Online]. Available: http://dx.doi.org/10.1016/j.envsoft.2015.12.011

[10] O. R. Sánchez Ccoyllo y C. G. Ordóñez-Aquino. Evaluación de la Calidad del Aire de Lima Metropolitana 2017. Senamhi, editor. Lima; Perú; 2017.

[11] European Union. CAQI Air quality index: Comparing Urban Air Quality across Borders, 2012. Available: http://www.airqualitynow.eu/download/CITEAIR-

Comparing_Urban_Air_Quality_across_Borders.pdf

\section{(c) (1)}

Los artículos publicados por TECNIA pueden ser compartidos a través de la licencia Creative Commons: CC BY 4.0. Permisos lejos de este alcance pueden ser consultados a través del correo revistas@uni.edu.pe 